\title{
Editorial
}

\section{Medical Editors Trial Amnesty (META)}

Reports of properly conducted randomised controlled trials are the foundation of safe and effective health care. However, a substantial proportion of all controlled trials never contribute to this knowledge base because they are not submitted for publication. ${ }^{12}$ This fact has a number of important implications for patient care. Firstly, underreporting of trials reduces the power of systematic reviews to detect moderate but clinically important treatment effects. As a result, patients may be denied effective forms of health care. Secondly, since trials that show more promising treatment effects are more likely to be submitted for publication, research syntheses based on published studies can give misleading conclusions about treatment effectiveness, thus exposing patients to useless or even harmful therapies. ${ }^{3}$ Finally, patients may be asked to participate in new research studies designed to address questions that have, in truth, already been answered. ${ }^{4}$

Trials go unreported for a myriad of reasons: the most common is that investigators think the results are "not interesting," and it is well documented that trials with nonsignificant results are substantially less likely to be submitted for publication. ${ }^{1}$ Sometimes participant recruitment takes longer than anticipated at the expense of time and resources set aside for report writing; investigators may change jobs with the result that important work remains unfinished; or investigators may discover a recently published trial on the same topic and conclude that their own results are now redundant. Editors must also take some of the responsibility. There is a limit to the number of reports we can publish and sometimes we are forced to decline publication. Many investigators regret not having published their trial results, and almost all investigators when contacted about unreported data are delighted to provide them.

Because of the important consequences of unreported trials, the editors of nearly 100 international medical journals have joined together to call an unreported trial amnesty. Although amnesty means giving pardon, we hope that investigators will see this as an opportunity-namely, to make the results of previously unreported trials publicly accessible, thus having the potential to contribute to the scientific foundation of health care. We urge all investigators with unreported trial data to register their trial or trials by filling in and returning an unreported trial registration form. We would like to register any unreported controlled trial, including trials that have only been published as an abstract. Registration can be undertaken by anyone able to provide the registration information, even if they are unable to provide the actual trial data. We expect a degree of duplicate registration. Registration information can be posted or faxed to the editorial offices of this journal. Alternatively, registration information can be sent by email (meta@ucl.ac.uk). The information will be made available by listing the trial details on a web site, and in other ways as appropriate. If specific trial data are required-for example, by those conducting systematic reviews, then the reviewer will be able to seek this information directly from the trialist. Some of the trials may be suitable for full publication, and the journal will be happy to consider these.

Medical editors are acutely aware of the trials and tribulations of research reporting. But on this occasion, because of the serious implications of unreported research, we are endeavouring to cleave the trials from the tribulations. We cannot confidently estimate the total number of unreported trials, but we are confident of a good response.

RICHARD SMITH Editor, $B M Y$ IAN ROBERTS Child Health Monitoring Unit, Institute of Child Health, London WC1N 1EH MOHSEN SHAHMANESH Editor, Genitourinary Medicine

Since the first notice of the amnesty was published in the $B M \mathcal{F}$ (13 Sept) we have received 25 notifications of unpublished trials

1 Dickersin K, Min YI. NIH clinical trials and publication bias. On-line $f$ Curr Clin Trials [serial on-line] 1993;Apr 28: Doc No 50.

2 Easterbrook PJ, Berlin JA, Gopalan R, Matthews DR. Publication bias in clinical research. Lancet 1991;337:867-72.

3 Egger M, Davey Smith G. Misleading meta-analysis. BMf 1995;310 $752-4$.

4 Savulescu J, Chalmers I, Blunt J. Are research ethics committees behaving unethically? Some suggestions for improving performance and accountability. BMF 1996;313:1390-3.

\section{Unreported trial registration form}

Register any controlled trial which has not been published in full, including trials that have only been published as an abstract. Registration can be undertaken by anyone able to provide the registration information, even if they are unable to provide the actual trial data. Please complete one form for each trial being registered.

Contact details

Surname:

Forename(s):

Postal address:

Phone (with regional codes):

Fax (with regional codes):

Email:

Trial details

Approximate number of participants in the trial:

Type of participants (eg, people with head injury, women at risk of breast cancer):

Type of intervention (eg, steroids versus placebo, annual mammography versus standard practice):

\section{versus}

